\title{
Hippocampal Contribution to Context Encoding across Development Is Disrupted following Early-Life Adversity
}

\author{
Hilary K. Lambert, ${ }^{1}$ Margaret A. Sheridan, ${ }^{2}$ CEelly A. Sambrook, ${ }^{3}$ Maya L. Rosen, ${ }^{1}$ Mary K. Askren, ${ }^{1}$ \\ and ${ }^{-K a t i e}$ A. McLaughlin ${ }^{1}$ \\ ${ }^{1}$ Department of Psychology, University of Washington, Seattle, Washington 98105, 2Department of Psychology, University of North Carolina, Chapel Hill, \\ North Carolina 27599, and ${ }^{3}$ Department of Radiology, University of Washington, Seattle, Washington 98105
}

Context can drastically influence responses to environmental stimuli. For example, a gunshot should provoke a different response at a public park than a shooting range. Little is known about how contextual processing and neural correlates change across human development or about individual differences related to early environmental experiences. Children $(N=60 ; 8-19$ years, 24 exposed to interpersonal violence) completed a context encoding task during fMRI scanning using a delayed match-to-sample design with neutral, happy, and angry facial cues embedded in realistic background scenes. Outside the scanner, participants completed a memory test for contextface pairings. Context memory and neural correlates of context encoding did not vary with age. Larger hippocampal volume was associated with better context memory. Posterior hippocampus was recruited during context encoding, and greater activation in this region predicted better memory for contexts paired with angry faces. Children exposed to violence had poor memory of contexts paired with angry faces, reduced hippocampal volume, and atypical neural recruitment on encoding trials with angry faces, including reduced hippocampal activation and greater functional connectivity between hippocampus and ventrolateral prefrontal cortex (vlPFC). Greater hippocampus-vlPFC connectivity was associated with worse memory for contexts paired with angry faces. Posterior hippocampus appears to support context encoding, a process that does not exhibit age-related variation from middle childhood to late adolescence. Exposure to dangerous environments in childhood is associated with poor context encoding in the presence of threat, likely due to greater vlPFC-dependent attentional narrowing on threat cues at the expense of hippocampus-dependent processing of the broader context.

Key words: childhood adversity; context; early-life stress; hippocampus; memory; violence

Significance Statement

The ability to use context to guide reactions to environmental stimuli promotes flexible behavior. Remarkably little research has examined how contextual processing changes across development or about influences of the early environment. We provide evidence for posterior hippocampus involvement in context encoding in youth and lack of age-related variation from middle childhood to late adolescence. Children exposed to interpersonal violence exhibited poor memory of contexts paired with angry faces and atypical neural recruitment during context encoding in the presence of threatening facial cues. Heightened attention to threat following violence exposure may come at the expense of encoding contextual information, which may ultimately contribute to pathological fear expressed in safe contexts.

\section{Introduction}

Humans constantly interact with complex contextual stimuli, including sights, sounds, and smells associated with particular

Received Aug. 17, 2016; revised Jan. 7, 2017; accepted Jan. 12, 2017.

Author contributions: H.K.L., M.A.S., and K.A.M. designed research; H.K.L., K.A.S., and M.K.A. performed research; H.K.L., K.A.S., M.L.R., and M.K.A. analyzed data; H.K.L., M.A.S., and K.A.M. wrote the paper.

This work was supported by a Brain and Behavior Foundation National Alliance for Research on Schizophrenia and Depression Young Investigator Grant, National Institute of Mental Health R01-MH103291, and a Jacobs Foundation Early Career Research Fellowship to K.A.M.

The authors declare no competing financial interests.

Correspondence should be addressed to Dr. Katie A. McLaughlin, Department of Psychology, University of Washington, Box 351525, Seattle, WA 98195. E-mail: mclaughk@uw.edu. locations. Context encoding involves forming an integrated representation of contextual stimuli and binding that representation with focal cues. Memory of contextual information from past encounters with a cue can facilitate accurate interpretation of that cue and appropriate responding. For example, a gunshot should provoke a different response at a public park than a shooting range. Remarkably little is known about how contextual processing and associated neural correlates vary across development or about individual differences related to early experience. Here we 
examine context encoding, the earliest phase of contextual processing.

The neural networks underlying context encoding are well characterized in rodents and adult humans. In rodents, the dorsal hippocampus supports context encoding (Phillips and LeDoux, 1992; Young et al., 1994; Maren and Fanselow, 1997) and shortterm storage of context memories (Kim and Fanselow, 1992; Anagnostaras et al., 1999). In adult humans, the parahippocampal gyrus and anterior hippocampus are recruited during implicit encoding of visual contexts paired with objects or faces (Hayes et al., 2007, 2010), consistent with research implicating these regions in associative learning more broadly (Davachi, 2006).

Scant research has examined developmental variation in neural recruitment during context encoding or in recall of contextcue pairings. We do so in the current study. We hypothesized that the hippocampus would be involved in context encoding in youth and that activation in this region would predict memory for contextual information. With regard to developmental variation, two patterns are possible. First, context encoding might be an implicit form of associative learning that remains stable across childhood and adolescence. Implicit learning emerges early in development and exhibits few age-related differences after early childhood (Meulemans et al., 1998; Vinter and Perruchet, 2000; Dixon et al., 2010). Alternatively, context encoding may resemble explicit forms of learning and memory, such as paired-associate learning and declarative memory, that improve with age. Memory of explicitly encoded scenes and stimulus pairings improves across childhood and adolescence, and associated hippocampal activation and functional connectivity also vary developmentally (Ghetti et al., 2010; Ofen et al., 2012; DeMaster and Ghetti, 2013).

Although information processing systems are tuned by early environmental experience to maximize adaptation, little is known about how environmental experience influences contextual processing. Threatening experiences early in life (e.g., interpersonal violence) influence emotional processing to facilitate rapid identification of signals of potential danger (McLaughlin et al., 2014; Sheridan and McLaughlin, 2014). For example, children exposed to violence exhibit heightened attention to angry faces (Pollak and Tolley-Schell, 2003; Shackman et al., 2007). We investigated whether childhood violence exposure influences context encoding.

Existing research suggests that childhood violence exposure could result in either general context encoding deficits or deficits specific to contexts in which threat is present. In support of the former hypothesis, animal models show that enhanced corticotropin-releasing hormone binding in the hippocampus following chronic stress reduces dendritic spines and branching in hippocampal neurons (Brunson et al., 2001; Ivy et al., 2010) and impairs hippocampus-dependent spatial memory (McLaughlin et al., 2007) and context-modulation of fear (Cohen et al., 2009). In humans, reduced hippocampal volume has been observed in maltreated children (Lim et al., 2014; Hanson et al., 2015; McLaughlin et al., 2016). These findings suggest the possibility of broad impairments in hippocampal function in children exposed to violence. Alternatively, childhood violence exposure may influence context encoding only in the presence of threat cues, as narrowing of attention on these cues (e.g., Pollak and TolleySchell, 2003; Shackman et al., 2007) may come at the expense of processing the broader context. We expected that children who experienced violence would exhibit poor retrieval of contextual information and reduced hippocampal recruitment during context encoding, and that deficits would be most pronounced in the presence of threat cues.

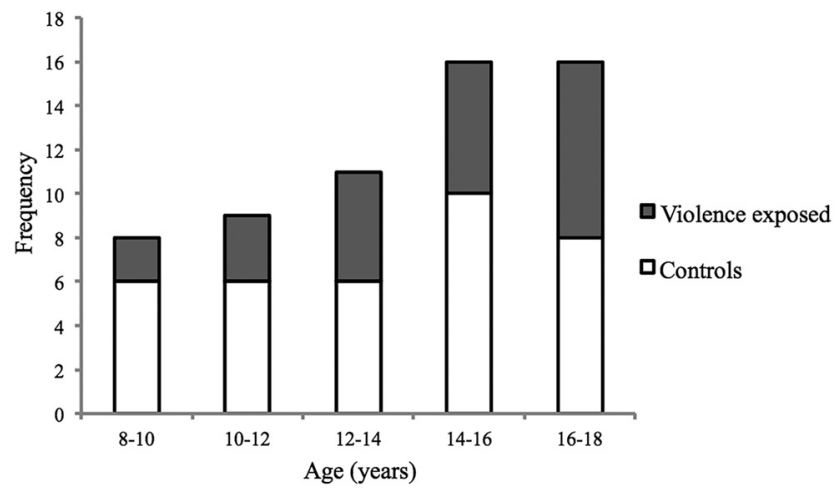

Figure 1. Age distribution of violence-exposed and control participants.

Table 1. Distribution of demographics by violence exposure $(N=60)$

\begin{tabular}{lcccc}
\hline & $\begin{array}{l}\text { Violence- } \\
\text { exposed } \\
(n=24)\end{array}$ & $\begin{array}{l}\text { Controls } \\
(n=36)\end{array}$ & $\chi^{2}$ & $p$ \\
\hline Female, \% ( $n)$ & $54.2(13)$ & $47.2(17)$ & 0.28 & 0.598 \\
Race/ethnicity, \% (n) & & & $13.03^{* *}$ & 0.011 \\
White, \% ( $n)$ & $37.5(9)$ & $69.4(25)$ & & \\
Black, \% $(n)$ & $25.0(6)$ & $0.0(0)$ & & \\
Hispanic, \% (n) & $25.0(6)$ & $13.9(5)$ & & \\
Asian/Pacific Islander, \% (n) & $8.3(2)$ & $13.9(5)$ & & \\
Biracial/other, \% (n) & $4.2(1)$ & $2.8(1)$ & & \\
Poverty, \% (n) & $54.2(13)$ & $16.7(6)$ & $10.27^{* *}$ & 0.001 \\
& & & $t$ & \\
\hline Age, mean (SD) & $14.44(3.01)$ & $13.63(2.99)$ & -1.02 & 0.312 \\
Emotional neglect, mean (SD) & $17.71(5.90)$ & $12.86(4.25)$ & $-3.70^{* *}$ & 0.0005 \\
IQ, mean (SD) & $96.29(13.27)$ & $111.86(13.06)$ & $4.50^{* *}$ & $<0.0001$ \\
Internalizing symptoms, mean (SD) & $57.04(9.50)$ & $46.08(10.60)$ & $-4.09^{* *}$ & 0.0001 \\
Externalizing symptoms, mean (SD) & $55.58(11.21)$ & $45.33(10.98)$ & $-3.51^{* *}$ & 0.001 \\
\hline
\end{tabular}

${ }^{* *} p<0.01$.

\section{Materials and Methods}

\section{Sample}

A sample of 60 children $8-19$ years of age (mean $\pm \mathrm{SD}, 13.95 \pm 3.00$ years) participated. Half of the sample was female $(n=30,50.0 \%)$. The sample was recruited in Seattle between February 2014 and February 2015. Youths were recruited at schools, after-school and prevention programs, medical clinics, and in the general community. To ensure variation in exposure to violence, recruitment targeted neighborhoods with high levels of violent crime, clinics that serve a predominantly low socioeconomic status area, and agencies that support families exposed to violence (e.g., domestic violence shelters and programs for parents mandated by Child Protective Services to receive intervention). Participants were screened out during recruitment based on the presence of a pervasive developmental disorder or any contraindication to MRI scanning. The Institutional Review Board at the University of Washington approved all procedures. Written informed consent was obtained from legal guardians, and youths provided written assent.

For demographic characteristics of the sample according to violence exposure, see Figure 1 and Table 1. Participants with violence exposure were matched to control participants on sex and age. However, violenceexposed participants were less likely to be white, had lower IQ, were more likely to be living in poverty, and had greater exposure to neglect than participants without a history of experiencing violence. Violenceexposed participants also had higher levels of internalizing and externalizing symptoms than control participants.

\section{Context encoding and memory task}

The task involved a context encoding phase in the scanner and a context memory phase outside the scanner (Fig. 2). During the encoding phase, participants completed a working memory task using a delayed match- 


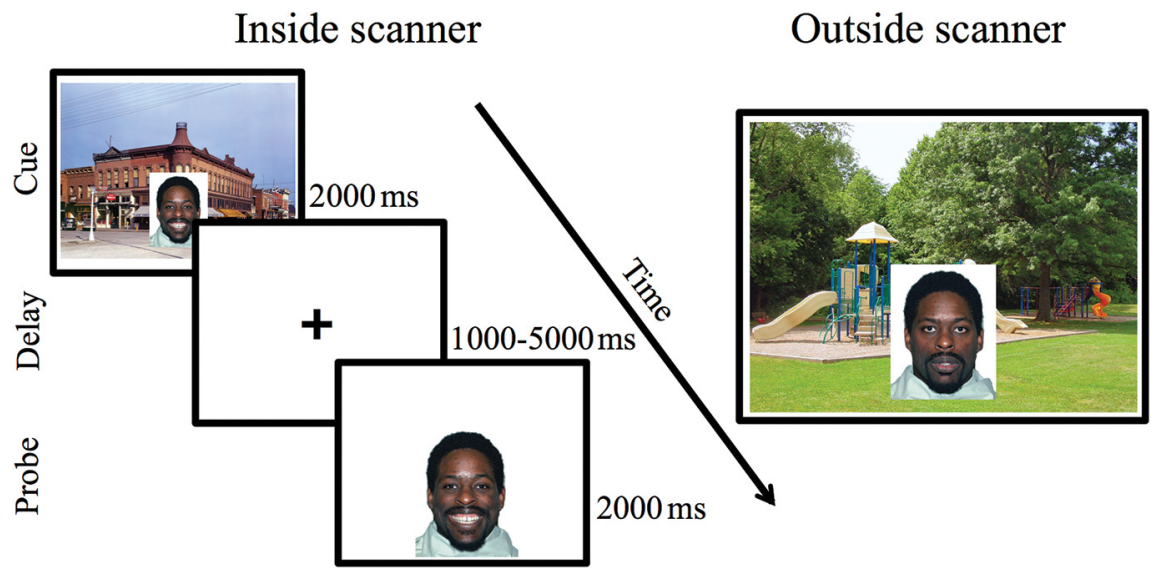

Figure 2. Context encoding task. Left, The context encoding phase occurred inside of the scanner. Right, The context memory phase occurred outside of the scanner.

to-sample design. The encoding phase occurred over two runs of 50 trials each, and each trial involved a cue (2000 ms), delay (1000-5000 ms), and probe $(2000 \mathrm{~ms})$. The cue involved emotional facial stimuli presented in realistic background scenes. Evidence from rodent studies suggests that the dorsal hippocampus is involved in context encoding only when context is in the background of more salient foreground cues, but not in the absence of focal cues (Phillips and LeDoux, 1994). To ensure that context encoding occurred without awareness, participants were instructed to attend to the faces and their expressions, but were not instructed to focus on the background scenes. The probe involved a facial stimulus without a background scene, and participants were asked to indicate whether the probe face matched the cue face. The intertrial interval (ITI) was jittered (500-2000 ms).

Facial stimuli were drawn from a standardized stimulus set (Tottenham et al., 2009) and included neutral, happy, and angry faces distributed evenly across trials and presented in a counterbalanced order across participants. Five different actors were used, each expressing a neutral, happy, and angry facial expression and each presented 6 or 7 times for each facial expression. Fifteen different contexts were used, including five outdoor street scenes, five outdoor nature scenes, and five indoor scenes. Each context type was consistently paired with an emotion type (e.g., all outdoor street scenes were paired with happy facial expressions, all outdoor nature scenes were paired with neutral facial expressions, and all indoor scenes were paired with angry facial expressions), and the specific context-emotion pairings were counterbalanced across participants. Pilot data suggested that context memory improved when contexts and faces were paired in this way during encoding.

During the context memory phase outside of the scanner, participants were presented with trials of faces embedded within a context. Participants were instructed to indicate whether the face was in the same context as seen during the encoding phase. There were 45 trials in total, including trials of context-face pairings that were accurate ( 15 trials) and trials of context-face pairings that were inaccurate (evenly distributed across three types with 15 trials each: familiar context with novel face, novel context with familiar face, and familiar context and face but inaccurate pairing). Three of each type of pairing (one for each emotion type) was presented for each of the five actors. Context memory accuracy was calculated by dividing the correct responses for all trials by the total number of trials, with a higher number indicating higher accuracy. Context memory accuracy was also calculated separately for trials involving facial cues with neutral, happy, and angry expressions.

\section{Violence exposure}

An interview and a self-report questionnaire were used to assess exposure to violence. Specifically, we assessed exposure to physical abuse, sexual abuse, or domestic violence, experiences that reflect a childhood environment characterized by threat. The Childhood Experiences of Care and Abuse (CECA) is an interview that assesses multiple aspects of care- giving experiences (Bifulco et al., 1997). We used the CECA to assess physical abuse, sexual abuse, and witnessing domestic violence (i.e., directly observing violence directed at a caregiver). Inter-rater reliability for CECA maltreatment reports is excellent, and multiple validation studies suggest high agreement between siblings on reports of maltreatment (Bifulco et al., 1997). We also administered the Childhood Trauma Questionnaire, a selfreport questionnaire that assesses the frequency of child maltreatment exposure, including physical and sexual abuse, and has sound psychometric properties (Bernstein et al., 1997). Participants were classified as violence-exposed whether they reported physical or sexual abuse or witnessing more than two incidents of domestic violence on the CECA or whether they received a score on the Childhood Trauma Questionnaire physical and sexual abuse subscales above a validated threshold (Walker et al., 1999). A total of $40.0 \%$ of the sample $(n=24)$ was violence-exposed based on this definition.

\section{Potential confounding variables}

Poverty. A parent or guardian completed a socioeconomic status measure. The income-to-needs ratio was calculated by dividing total household income by the 2015 U.S. Census-defined poverty line for a family of that size, with a value $<1$ indicating that a family was living below the poverty line.

Emotional neglect. The CECA includes a self-report questionnaire that assesses parental neglect. Scores on neglect items were summed for each parent figure separately. Summed scores on paternal and maternal neglect subscales were averaged to produce an overall neglect score for each participant. Higher scores indicated greater exposure to neglect.

IQ. The Wechsler Abbreviated Scale of Intelligence, Edition 2 (Wechsler, 1999) was used to estimate IQ, with higher scores indicating higher intellectual ability.

Psychopathology. Participants completed the Youth Self Report (YSR), a measure of internalizing and externalizing symptoms (Achenbach, 1991). The YSR scales are among the most widely used measures of youth emotional and behavioral problems and use extensive normative data to generate age-standardized estimates of symptom severity. Higher scores indicate worse symptom severity. Symptom scores on the Child Behavior Checklist (Achenbach, 1991), the parent-report version of the YSR, were used for two participants with missing YSR data.

\section{Image acquisition and processing}

Scanning was performed on a 3T Phillips Achieva scanner at the University of Washington Integrated Brain Imaging Center using a 32-channel head coil. T1-weighted multiecho MPRAGE volumes were acquired $\left(\mathrm{TR}=2530 \mathrm{~ms}, \mathrm{TE}=1640-7040 \mu \mathrm{s}\right.$, flip angle $=7^{\circ}, \mathrm{FOV}=256 \mathrm{~mm}^{2}$, 176 slices, in-plane voxel size $=1 \mathrm{~mm}^{3}$ ). BOLD signal during functional runs was acquired using a gradient-echo $\mathrm{T} 2{ }^{*}$-weighted EPI sequence. Thirty-two 3-mm-thick slices were acquired parallel to the AC-PC line $\left(\mathrm{TR}=2000 \mathrm{~ms}, \mathrm{TE}=25 \mathrm{~ms}\right.$, flip angle $=79^{\circ}$, bandwidth $=2040 \mathrm{~Hz}$, echo spacing $=0.629 \mathrm{~ms}$, FOV $=224 \times 224$, matrix size $=76 \times 74$ ). Before each scan, four images were acquired and discarded to allow longitudinal magnetization to reach equilibrium.

Neural structure. T1-weighted scans were processed using FreeSurfer version 5.3 (Fischl and Dale, 2000). Automatic image segmentation was used to identify subcortical gray matter structures. The results were inspected and manually edited to optimize accurate placement of gray/ white and gray/CSF borders. Subcortical segmentation was used to measure hippocampal volume.

Neural function. Preprocessing and statistical analysis of fMRI data was performed in FSL (Jenkinson et al., 2012) and included spatial realignment, simultaneous motion and slice-time correction (Roche, 2011), 
and spatial smoothing ( $6 \mathrm{~mm}$ FWHM). Data were inspected for artifacts, and volumes with motion $>2 \mathrm{~mm}$ or $>3$ SD change in signal intensity were excluded from analysis. Six rigid-body motion regressors were included in person-level models. Person- and group-level models were estimated in FSL. A component-based anatomical noise correction method (Behzadi et al., 2007) was used to reduce noise associated with physiological fluctuations. Following estimation of person-level models, the resulting contrast images were normalized into standard space, and anatomical coregistration of the functional data with each participant's T1-weighted image was performed using FSL. Normalization was implemented in Advanced Normalization Tools software, version 2.1.0 (Avants et al., 2011).

\section{Statistical analysis}

An outlier analysis was conducted for all behavioral and neural outcome variables with a criterion of $3 \mathrm{SDs}$ above or below the group mean. Only one participant, who was a control, exhibited hippocampal activation during context encoding $>3$ SDs below the mean. Analyses involving hippocampal function were conducted both with and without this outlier.

Context memory. Linear regression was used to examine associations of age, sex, hippocampal volume, and hippocampal BOLD signal during context encoding (Cue > ITI) with context memory. Linear, logarithmic, and quadratic age effects were estimated. We also examined age $\times$ hippocampal volume and age $\times$ hippocampal BOLD signal interactions. A univariate ANOVA was used to examine context memory based on violence exposure. Variation in context memory by emotion condition was examined with a repeated-measures ANOVA with emotion of the facial cue (neutral, happy, and angry) as a within-subjects factor. Age, sex, violence exposure, and hippocampal volume were added to these models as between-subjects factors. Significant interactions with emotion were followed up with univariate ANOVAs for neutral, happy, and angry conditions, separately. Associations of hippocampal BOLD signal during context encoding with context memory were examined separately by emotion condition.

Neural function. fMRI data processing was performed using FEAT (fMRI Expert Analysis Tool) version 6.00, part of FSL (fMRIB's Software Library, www.fmrib.ox.ac.uk/fsl). Regressors were created by convolving a boxcar function of phase duration and amplitude one with the standard hemodynamic response function for each phase of the task (cue, delay, and probe) separately for neutral, happy, and angry facial cues. A GLM was constructed for each participant. Individual-level estimates of BOLD activity were submitted to group-level random effects models. Higherlevel analyses were performed using FLAME (fMRIB's Local Analysis of Mixed Effects) Stage 1 (Beckmann et al., 2003; Woolrich et al., 2004; Woolrich, 2008). To examine neural recruitment during context encoding, we examined BOLD activity during the cue period only (Cue $>$ ITI). A whole-brain analysis was conducted to identify clusters associated with context encoding in the entire sample. We used a conservative approach to cluster-level correction that is not associated with elevated risk of false-positive findings (Eklund et al., 2016). Specifically, we applied stringent cluster-level correction in FSL (voxel-level threshold of $z>2.3, p<$ 0.01 ; cluster-level threshold of $z>3.0, p<0.001$ ) to our models run in FSL FLAME.

We additionally used a region of interest (ROI) analysis to examine hippocampal activation. Rodent and adult human research differs on which subregion of the hippocampus is recruited during context encoding (Phillips and LeDoux, 1992; Young et al., 1994; Maren and Fanselow, 1997; Hayes et al., 2007, 2010). Without an a priori hypothesis of which subregion would be involved, we used an empirical approach. Specifically, an ROI was created by masking functional activation during context encoding (Cue > ITI) from the whole-brain analysis in the entire sample with a structural mask of the hippocampus from the HarvardOxford Subcortical Atlas in FSL (20\% threshold; separately for right and left hemispheres). The functional mask was created based on 58 subjects who had two runs of data (two participants completed only one run). This approach isolated the portion of the hippocampus that was significantly active during context encoding in our sample. ROI analyses included all 60 participants. Parameter estimates for each trial type (any facial cue and neutral, happy, and angry facial cues) were extracted for each participant using FSL. Because the pattern of associations of hippocampal activation with task performance, age, and violence exposure was similar in direction and magnitude in the right and left hemispheres, we present all analyses with a bilateral hippocampus ROI constructed by averaging the parameter estimates from the right and left hemispheres.

We examined variation in hippocampal activation during context encoding as a function of age using linear regression; linear, logarithmic, and quadratic age effects were estimated. Univariate ANOVA was used to examine hippocampal activation during context encoding as a function of violence exposure, and repeated-measures ANOVA with emotion as a within-subjects factor was used to examine violence $X$ emotion and age $\times$ emotion interactions. Interactions were followed up with individual univariate ANOVAs for neutral, happy, and angry conditions. Covariates for age and sex were not included as violence-exposed and control participants were matched on age and sex, and these factors had no influence on hippocampal activation.

Task-based functional connectivity. We conducted a whole-brain psychophysiological interaction (PPI) analysis to identify violence-related differences in functional connectivity of the hippocampus with other brain regions during context encoding (O'Reilly et al., 2012). PPI was only conducted for emotion conditions where significant group differences emerged in behavior and neural function (i.e., the angry condition). We extracted the mean BOLD signal time-series within right and left hippocampal ROI seed regions. Hippocampal vectors were multiplied by the task condition of interest (angry facial cue $>$ ITI) and then convolved with the canonical hemodynamic response function to form the PPI vector. We applied the same conservative cluster-level correction as in the whole-brain analysis (voxel-level threshold of $z>2.3, p<0.01$; cluster-level threshold of $z>3.0, p<0.001$ ).

As described below, participants exposed to violence exhibited significantly greater functional connectivity between right hippocampus and right ventrolateral prefrontal cortex (vlPFC) during context encoding on angry trials than control participants. In our final analysis, we examined whether the degree of functional connectivity between these two regions was associated with context memory accuracy on trials involving angry faces. To test this, we extracted the correlation between hippocampus and vlPFC activation, specifically during angry trials, for each participant to produce subject-level estimates of functional connectivity. This was accomplished by extracting the parameter estimate for the PPI regressor (i.e., the interaction of the hippocampal BOLD signal time-series and the task regressor for angry trials) from a vlPFC ROI for each participant. Linear regression was used to examine the association of subject-level hippocampus-vlPFC seed-to-seed correlations with performance on angry trials of the context memory test.

We constructed the vlPFC ROI for this analysis based on MNI coordinates reported in a prior study on attention to threat in adolescents (Monk et al., 2006). We used this approach because significant hippocampus-vlPFC connectivity emerged only in the PPI analysis comparing children with violence exposure to controls. Using this mask to construct the vlPFC ROI for analysis with the behavioral data would have increased the likelihood of false positive findings because violence exposure was associated both with hippocampus-vlPFC connectivity and context memory accuracy. The bias associated with this type of double-dipping used to define ROIs in fMRI studies is well established (Vul et al., 2009). As such, we used a more conservative approach that avoids this bias by using the coordinates from a related study to create the vlPFC ROI (Vul et al., 2009). We selected a study on attention to threat that included a sample of a similar age range as the present study, that used similar stimuli (i.e., angry faces), and that observed right vlPFC activation in response to angry faces (Monk et al., 2006). We drew a sphere with a $5 \mathrm{~mm}$ radius around the MNI coordinates of this vlPFC activation $(x, y, z=42,39,-17)$.

Mediation. We examined whether the association of violence exposure with context memory accuracy was explained by variation in hippocampal volume, activation, and functional connectivity with the vlPFC. We used a standard test of statistical mediation to test significance of indirect effects using a bootstrapping approach that provides confidence intervals for the indirect effects (Hayes, 2013). 


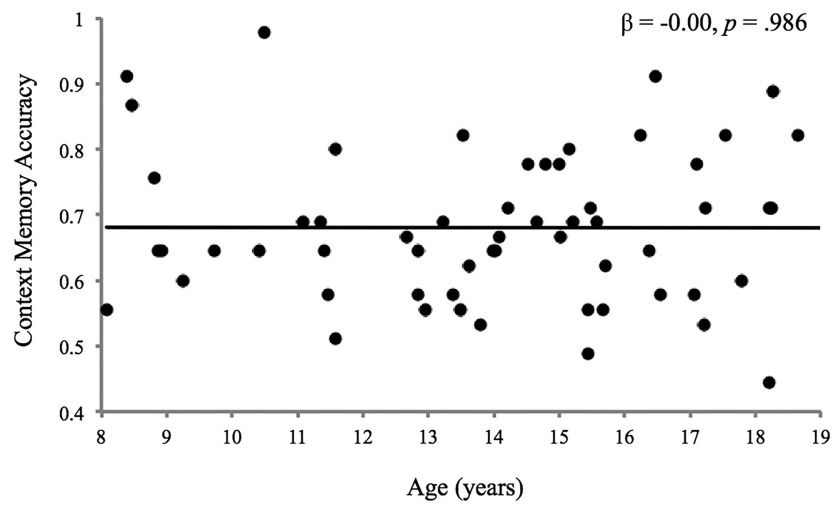

Figure 3. Age and context memory accuracy on trials involving all facial targets.

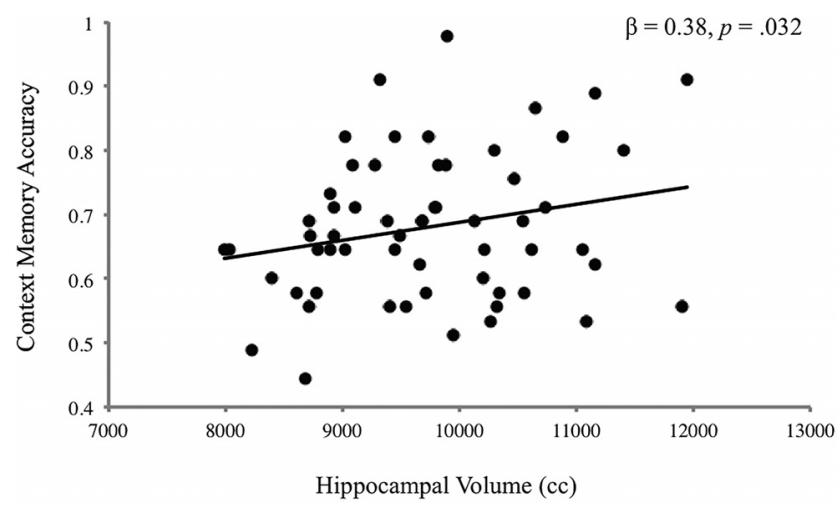

Figure 4. Bilateral hippocampal volume and context memory accuracy on trials involving all facial targets, adjusting for age, sex, and total brain volume.

Specificity to violence exposure. Several socio-demographic and mental health characteristics differed between the violence-exposed and control groups, consistent with extensive prior research on early-life adversity. To determine whether these factors were potential confounders of the association between violence exposure and our outcomes, we first examined whether these factors were associated with context memory accuracy, hippocampal volume, hippocampal BOLD signal, and whole-brain functional connectivity. We controlled for any characteristics that were associated with one of our outcomes of interest in our analyses of violence exposure.

\section{Results}

\section{Context memory}

Context memory performance was above chance $(68.1 \pm 11.45 \%)$. Accuracy did not vary by emotion condition $\left(F_{(2,116)}=0.16, p=\right.$ $0.85)$. Age and sex were not associated with context memory overall or by emotional condition ( $p$ values $=0.11-0.99$; Fig. 3 ). Larger bilateral hippocampal volume predicted higher context memory accuracy, controlling for age, sex, and total brain volume $(\beta=0.38$, $p=0.032$; Fig. 4 ), and this association did not vary by age, sex, or emotion condition $(p$ values $=0.41-0.60)$.

\section{Neural activation during context encoding}

Whole-brain analysis revealed four clusters of activation during context encoding (Cue > ITI) (Table 2; Fig. 5). The first cluster spanned bilateral occipital, parietal, and temporal cortex, including the posterior parahippocampal gyrus, fusiform gyrus, precuneus, posterior cingulate, and extending into inferior temporal gyrus, posterior hippocampus, and thalamus. Two clusters in PFC included right and left middle frontal gyrus. On the medial surface, one cluster included the bilateral anterior cingulate cortex/supplementary motor area.
Table 2. Whole-brain/whole-group analysis ${ }^{a}$

\begin{tabular}{|c|c|c|c|c|c|c|}
\hline \multirow[b]{2}{*}{ Anatomical region } & \multirow[b]{2}{*}{$x$} & \multirow[b]{2}{*}{$y$} & \multirow[b]{2}{*}{$z$} & \multirow[b]{2}{*}{ Voxels } & \multicolumn{2}{|c|}{ Maximum } \\
\hline & & & & & Zscore & $p$ \\
\hline Lingual gyrus $(\mathrm{R}, \mathrm{L})$ & 6 & -84 & -6 & 21,208 & 11 & $<0.0001$ \\
\hline Fusiform gyrus $(\mathrm{R}, \mathrm{L})$ & - & - & - & - & - & - \\
\hline Intraparietal sulcus (R, L) & - & - & - & - & - & - \\
\hline Lateral occipital cortex (R, L) & - & - & - & - & - & - \\
\hline Intracalcarine cortex (R, L) & - & - & - & - & - & - \\
\hline Cerebellum (R, L) & - & - & - & - & - & - \\
\hline Precuneus cortex (R, L) & - & - & - & - & - & - \\
\hline Inferior temporal gyrus (R, L) & - & - & - & - & - & - \\
\hline Posterior parahippocampal gyrus $(\mathrm{R}, \mathrm{L})$ & - & - & - & - & - & - \\
\hline Posterior cingulate gyrus (R, L) & - & - & - & - & - & - \\
\hline Supracalcarine cortex $(\mathrm{R}, \mathrm{L})$ & - & - & - & - & - & - \\
\hline Hippocampus (R, L) & - & - & - & - & - & - \\
\hline Superior parietal lobule $(\mathrm{R}, \mathrm{L})$ & - & - & - & - & - & - \\
\hline Thalamus (R, L) & - & - & - & - & - & - \\
\hline Middle frontal gyrus (L) & -40 & 6 & 36 & 1001 & 4.77 & $<0.0001$ \\
\hline Precentral gyrus (L) & - & - & - & - & - & - \\
\hline Inferior frontal gyrus (L) & - & - & - & - & - & - \\
\hline Inferior frontal gyrus (R) & 38 & 10 & 22 & 713 & 4.96 & $<0.0001$ \\
\hline Middle frontal gyrus (R) & - & - & - & - & - & - \\
\hline Precentral gyrus (R) & - & - & - & - & - & - \\
\hline Paracingulate gyrus (R, L) & -8 & 12 & 52 & 413 & 6.38 & $<0.0001$ \\
\hline Superior frontal gyrus (R, L) & - & - & - & - & - & - \\
\hline Supplementary motor cortex $(\mathrm{R}, \mathrm{L})$ & - & - & - & - & - & - \\
\hline
\end{tabular}

${ }^{a}$ Regions of the brain that were significantly active during context encoding (any facial cue $>$ ITI) in the entire sample $(N=60)$. Cluster-level correction applied in FSL. $z>2.3, p<0.01$ was the voxel-level threshold. $z>3.0$, $p<0.001$ was the cluster-level threshold. Laterality of regions is specified: $R$, Right; $L$, left.

Age was not associated with hippocampal activation during context encoding ( $p$ values $=0.27-0.95)$, and no age $\times$ emotion interaction was observed $\left(F_{(2,114)}=0.96, p=0.39\right)$.

\section{Brain-behavior associations}

The hippocampus ROI encompassed posterior hippocampus, which was the only portion of the hippocampus that was active during context encoding in the whole-brain analysis of the entire sample.

Greater bilateral hippocampal BOLD signal during context encoding was associated with higher context memory accuracy specifically on trials with angry facial cues $(\beta=0.28, p=0.030$; Fig. 6), but not overall or for happy or neutral trials ( $p$ values $=$ $0.52-0.75)$. There was no interaction between age and hippocampal BOLD signal during encoding on context memory overall or for angry, happy, or neutral trials ( $p$ values $=0.45-$ $0.64)$. Results were unchanged when the single outlier was excluded.

\section{Violence exposure and context memory}

Violence exposure was unrelated to overall context memory $\left(F_{(1,57)}=2.99, p=0.09\right)$. However, a significant interaction emerged between violence exposure and emotion in predicting context memory $\left(F_{(2,114)}=4.32, p=0.016\right.$; Fig. 7$)$. Violence exposure was associated with context memory on trials involving angry facial cues $\left(F_{(1,57)}=9.88, p=0.003\right)$, but not on trials involving happy or neutral facial cues ( $p$ values $=0.24-0.99$ ). Specifically, participants exposed to violence exhibited lower context memory accuracy for contexts paired with angry facial targets $(61.11 \pm 0.12)$ than control participants $(71.81 \pm 0.13)$.

\section{Violence exposure and neural structure}

Violence exposure was associated with bilateral hippocampal volume, controlling for age, sex, and total brain volume $\left(F_{(1,55)}=\right.$ $6.06, p=0.017$ ). Specifically, participants exposed to violence 

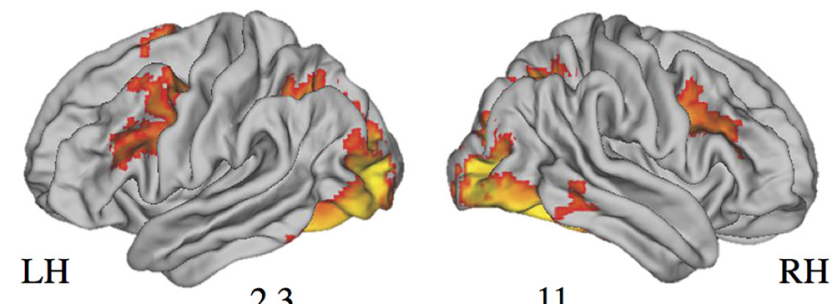

2.3

11

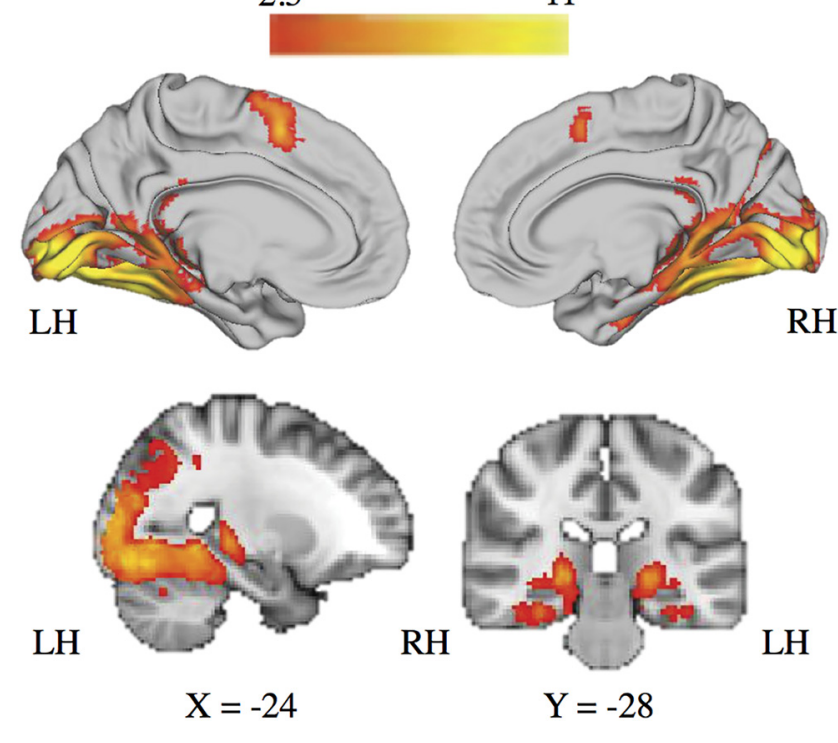

Figure 5. Regions of the brain with significant BOLD activation during context encoding (any facial cue $>I T I)$ in the entire sample $(N=60)$. Cluster-level correction applied in FSL. $z>2.3$, $p<0.01$ was the voxel-level threshold. $z>3.0, p<0.001$ was the cluster-level threshold.

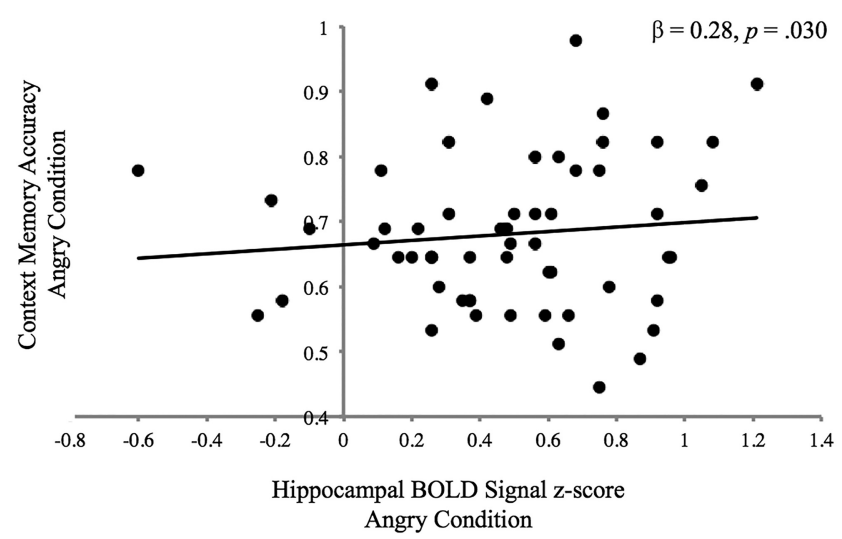

Figure 6. Bilateral posterior hippocampal BOLD signal during context encoding (angry facial cue $>$ ITI) and context memory accuracy on trials involving angry facial targets.

had smaller average hippocampal volume $(9265.8 \pm 775.1)$ than control participants $(10016.1 \pm 922.6)$.

\section{Violence exposure and neural function}

In ROI analysis, violence exposure was unrelated to hippocampal activation during context encoding overall $\left(F_{(1,57)}=1.78, p=\right.$ $0.19)$. However, an interaction between violence exposure and emotion was observed $\left(F_{(2,114)}=3.51, p=0.033\right.$; Fig. 8). Violence exposure was associated with hippocampal BOLD signal during context encoding in the presence of angry facial cues $\left(F_{(1,57)}=4.92, p=0.030\right)$, but not in the presence of neutral or happy facial cues ( $p$ values $=0.43-0.71$ ). Specifically, participants exposed to violence exhibited less hippocampal activation

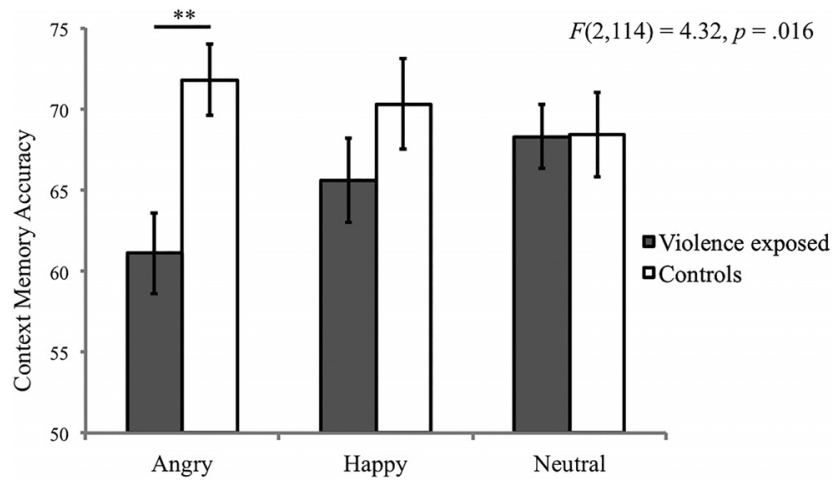

Figure 7. Interaction between violence exposure and emotion condition on context memory accuracy.

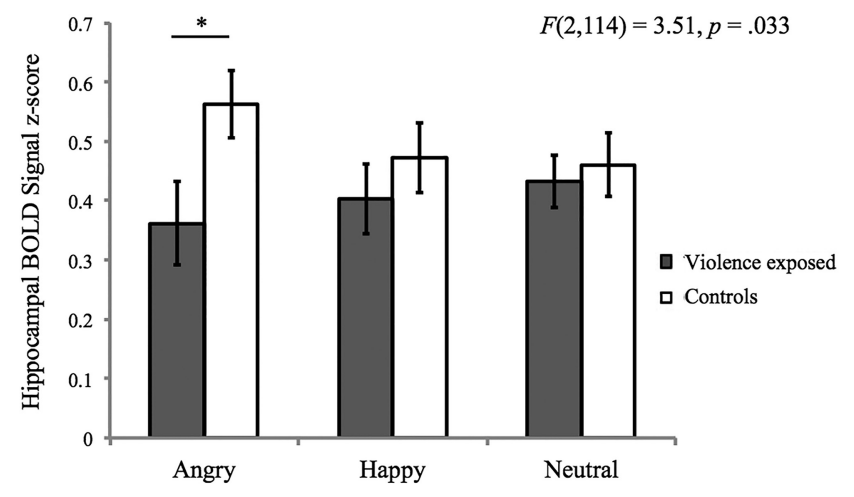

Emotion of Facial Cue Present During Context Encoding

Figure 8. Interaction between violence exposure and emotion condition on bilateral posterior hippocampal BOLD signal during context encoding.

during context encoding on angry trials $(0.36 \pm 0.35)$ than control participants $(0.56 \pm 0.34)$.

When the single outlier was removed, the violence by emotion interaction was unchanged $\left(F_{(2,112)}=3.46, p=0.035\right)$. However, an association of violence exposure with hippocampal BOLD signal during context encoding (regardless of emotion) emerged $\left(F_{(1,56)}=4.31, p=0.043\right)$. Specifically, less hippocampal BOLD signal during all trials of context encoding was observed in participants exposed to violence $(0.47 \pm 0.28)$ versus control participants $(0.62 \pm 0.28)$.

\section{Functional connectivity}

Whole-brain PPI analysis revealed that control participants had greater functional connectivity of right hippocampus with left middle frontal gyrus and left intraparietal sulcus during context encoding on angry trials compared with participants exposed to violence (Table 3; Fig. 9). Violence-exposed participants exhibited greater functional connectivity between right hippocampus and right vlPFC than control participants (Table 3; Fig. 9).

\section{Functional connectivity-behavior associations}

Greater functional connectivity between right hippocampus and right vlPFC during context encoding on trials with angry faces predicted worse memory for contexts paired with angry faces $(\beta=-0.49, p=0.0001$; Fig. 10$)$. This association remained when controlling for violence exposure $(\beta=-0.41$, $p=0.001)$. 
Table 3. Functional connectivity analysis by group ${ }^{a}$

\begin{tabular}{|c|c|c|c|c|c|c|c|}
\hline Posterior hippocampal seed region & Anatomical region & $x$ & $y$ & $z$ & Voxels & Maximum Zscore & $p$ \\
\hline \multicolumn{8}{|l|}{ Controls $>$ violence-exposed } \\
\hline \multirow[t]{5}{*}{$\mathrm{R}$} & Fusiform gyrus (L) & -28 & -72 & -16 & 140 & 3.98 & 0.010 \\
\hline & $\begin{array}{l}\text { Temporal occipital fusiform Cortex (L) } \\
\text { Cerebellum (L) }\end{array}$ & & & & & & \\
\hline & Precentral gyrus (L) & -46 & -8 & 52 & 133 & 3.48 & 0.014 \\
\hline & Middle frontal gyrus (L) & & & & & & \\
\hline & $\begin{array}{l}\text { Lateral occipital cortex (L) } \\
\text { Intraparietal sulcus (L) }\end{array}$ & -32 & -72 & 24 & 117 & 3.36 & 0.031 \\
\hline L & - & & & & & & \\
\hline \multicolumn{8}{|l|}{ Violence-exposed $>$ controls } \\
\hline $\mathrm{R}$ & Ventrolateral prefrontal cortex/frontal pole (R) & 24 & 56 & -4 & 149 & 3.41 & 0.006 \\
\hline L & - & - & - & - & - & - & - \\
\hline
\end{tabular}

${ }^{a}$ Significantly different clusters exhibiting functional connectivity with the right and left posterior hippocampus during context encoding (angry facial cues $>$ ITI) for participants exposed to violence versus control participants. (luster-leve correction applied in FSL. $z>2.3, p<0.01$ was the voxel-level threshold, and $z>3.0, p<0.001$ was the cluster-level threshold. Laterality of regions is specified: $R$, Right; L, left.

\section{Controls >}

Violence exposed
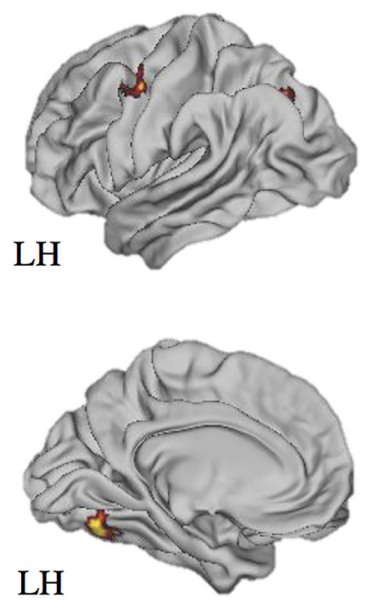

2.3
Violence exposed > Controls

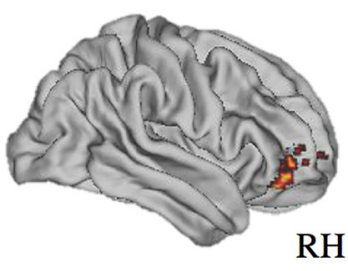

$\mathrm{RH}$

Figure 9. Regions of the brain with significantly different functional connectivity with the right posterior hippocampus during context encoding (angry facial cue $>$ ITI) for participants exposed to violence and control participants. Cluster-level correction applied in FSL. $z>2.3$, $p<0.01$ was the voxel-level threshold. $z>3.0, p<0.001$ was the cluster-level threshold.

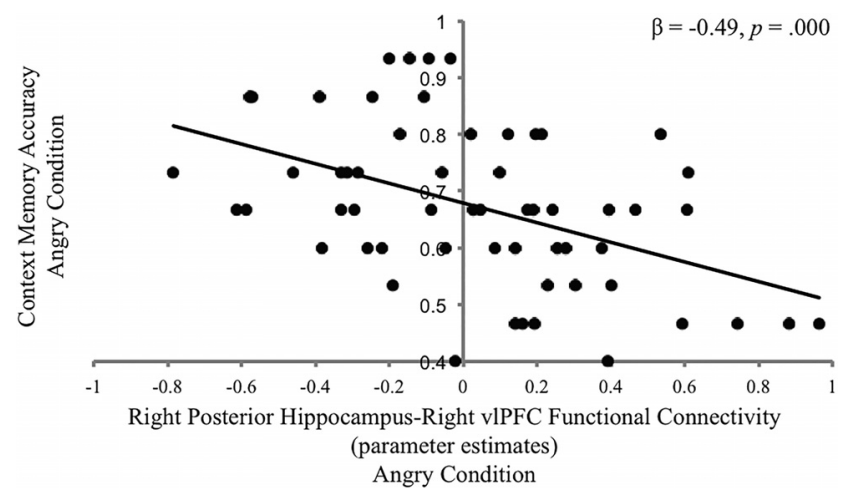

Figure 10. Functional connectivity between the right posterior hippocampus and right VIPFC during context encoding (angry facial cue $>$ ITI) and context memory accuracy on trials involving angry facial targets.

\section{Mediation}

We next evaluated whether the association of violence exposure with context memory accuracy on angry trials was explained by reduced hippocampal volume, reduced hippocampal activation during context encoding, and increased right hippocampus-right vlPFC functional connectivity during context encoding. Hippocampus-vlPFC functional connectivity mediated the association of violence exposure with context memory on angry trials (95\% CI: -0.078 to -0.008 ), as did hippocampal volume ( $95 \% \mathrm{CI}:-0.067$ to -0.003$)$. There was no indirect effect of violence exposure on context memory through hippocampal BOLD signal during context encoding.

\section{Specificity to violence exposure}

We tested whether characteristics that differed between the violence-exposed and control groups (poverty, neglect, IQ, internalizing symptoms, and externalizing symptoms) were potential confounders of the association of violence exposure with our four behavioral and neural outcomes (i.e., context memory accuracy on angry trials, hippocampal volume, hippocampal BOLD signal during context encoding on angry trials, and hippocampusvlPFC functional connectivity during context encoding on angry trials). To do so, we first examined whether these potential confounders were associated with each of the behavioral and neural outcomes of interest.

Neglect, internalizing symptoms, and externalizing symptoms were unrelated to all outcome variables. None of the potential confounders was associated with performance on the context memory test or with hippocampus-vlPFC functional connectivity.

Poverty was associated with one of the four outcomes. A significant interaction emerged between poverty and emotion condition in predicting hippocampal BOLD signal during context encoding, such that poverty was associated with lower hippocampal BOLD signal on angry trials $\left(F_{(1,50)}=4.31, p=0.043\right)$, but not on neutral or happy trials. Poverty was unrelated to context memory accuracy, hippocampal volume, and hippocampusvlPFC functional connectivity.

IQ was associated with two of the four outcomes. IQ was positively associated with hippocampal volume $\left(F_{(1,55)}=7.01\right.$, $p=0.011)$, and an IQ $\times$ emotion interaction emerged in predicting hippocampal BOLD signal, such that IQ was positively associated with hippocampal BOLD signal on angry trials $\left(F_{(1,57)}=\right.$ $4.14, p=0.047)$, but not on neutral or happy trials. IQ was unrelated to context memory accuracy and hippocampus-vlPFC functional connectivity.

In a final analysis, we evaluated whether the association of violence exposure with hippocampal volume and hippocampal BOLD signal during context encoding on angry trials persisted after adjustment for poverty and IQ. Associations of violence exposure, poverty, and IQ were all nonsignificant in these mod- 
els, likely due to high collinearity among these factors in our sample. After adjusting for IQ, the indirect effect of trauma exposure on task performance through hippocampal volume was no longer significant ( $95 \%$ CI: -0.049 to 0.003 ).

Hippocampus-vlPFC functional connectivity was unrelated to all confounders and was the sole mediator of trauma-related differences in task performance that remained significant in our final model.

\section{Discussion}

Remarkably little is known about how contextual processing and associated neural correlates vary across development and whether early-life experiences influence contextual processing. Our study demonstrates that the posterior hippocampus is involved in context encoding in youth and that context encoding does not change with age from middle childhood to late adolescence. Specifically, we found that memory for contextual information, hippocampal activation during context encoding, and associations of hippocampal activation with context memory did not change after age 8 years. In contrast, environmental experiences during childhood were associated with contextual encoding and retrieval. Specifically, children raised in threatening environments exhibited worse contextual memory and atypical neural recruitment during context encoding in the presence of threat cues.

We observed activation in the bilateral posterior hippocampus in a whole-brain analysis of context encoding. Greater posterior hippocampal activation during encoding was associated with better memory for contexts paired with angry facial cues, and larger hippocampal volume was associated with better context memory for all trial types. These findings are consistent with evidence for dorsal hippocampal involvement in context encoding in rodents (Phillips and LeDoux, 1992; Young et al., 1994; Maren and Fanselow, 1997), but not with evidence for anterior hippocampal involvement in context encoding in adult humans (Hayes et al., 2007, 2010). It is possible that anterior and posterior portions of the hippocampus contribute differently to context encoding across development. Indeed, posterior hippocampus increases in volume with age, whereas anterior hippocampus decreases (Gogtay et al., 2006). Posterior hippocampal activation supports retrieval of explicitly encoded stimulus pairings in childhood, whereas anterior hippocampal activation supports retrieval in adulthood (DeMaster and Ghetti, 2013). Future research is needed to determine whether a similar developmental posterior-anterior shift occurs for context encoding.

The fact that hippocampal activation during context encoding was associated with context memory only in the presence of angry faces is consistent with rodent work indicating that hippocampus involvement in context encoding increases in the presence of threat cues (Phillips and LeDoux, 1994). In humans, context memory is enhanced when encoding occurs in the presence of negative facial cues (Barrett and Kensinger, 2010). These results suggest that threat cues might enhance processing of the environment, potentially to facilitate avoidance of future threats. Given that context memory did not vary across emotion conditions in the current study and that the hippocampus was activated during context encoding in the presence of all facial cues, future research is needed to clarify whether the valence of foreground cues modulates contextual processing.

Performance on the context memory test and hippocampal activation during context encoding did not vary with age. Associations of hippocampal structure and function with context memory also did not vary with age. The lack of developmental variation suggests that context encoding emerges early in development. A change in context impairs explicit memory in 6-month-olds (Hayne et al., 2000; Robinson and Pascalis, 2004), suggesting that context encoding may be intact as early as infancy. The absence of explicit instruction to attend to background context in our task and the lack of developmental variation in context encoding and retrieval suggest that context encoding may occur implicitly. Implicit learning does not change after early childhood (Meulemans et al., 1998; Vinter and Perruchet, 2000; Dixon et al., 2010), whereas retrieval of explicitly encoded scenes and stimulus pairings and associated hippocampal correlates vary developmentally (Ghetti et al., 2010; Ofen et al., 2012; DeMaster and Ghetti, 2013). Future research examining context encoding and retrieval in a younger sample is needed to evaluate agerelated variation earlier in development.

Exposure to violence influenced context retrieval and neural correlates of context encoding, suggesting influences of early experience on contextual processing. Children exposed to violence had worse memory for contexts paired with angry faces, but not happy or neutral faces, than children who had never experienced violence. Children exposed to violence had smaller hippocampal volume and less hippocampal activation during encoding on trials involving angry faces than children without violence exposure. These findings replicate prior findings of reduced hippocampal volume in maltreated children (Lim et al., 2014; Hanson et al., 2015; McLaughlin et al., 2016) and extend this work by documenting atypical hippocampal function during context encoding following childhood violence exposure.

Why were neural and behavioral differences related to violence exposure specific to contexts encoded in the presence of angry facial cues? Children exposed to violence might have had faster attention orienting and longer sustained attention to angry faces, limiting processing of the broader context. Enhanced threat detection is an adaptation that likely promotes survival in dangerous environments (van Marle et al., 2009); indeed, child abuse is associated with heightened attention to angry facial expressions (Pollak and Tolley-Schell, 2003; Shackman et al., 2007). An alternative explanation is that violence-exposed youth directed attention away from angry facial cues, precluding encoding of context-cue pairings. However, violence-exposed children exhibited better working memory for angry faces than for happy or neutral faces in this sample (Jenness et al., unpublished observations), indicating that attentional bias toward rather than away from threat cues is a more likely explanation for poor context encoding in the presence of threat. Consistent with this interpretation, we found greater right lateralized hippocampus-vlPFC functional connectivity during context encoding in the presence of angry facial cues in children exposed to violence. Greater functional connectivity between these regions predicted lower context memory accuracy on angry trials and mediated the association of violence exposure with context memory. The vlPFC is involved in effortful regulation of attention toward or away from threat cues (Bishop, 2008; Shiba et al., 2016), and the right vlPFC is specifically associated with attention to threat in youth (Monk et al., 2006, 2008; Telzer et al., 2008). Anatomical connections between vlPFC and perirhinal and parahippocampal cortex have been identified in primates (Petrides and Pandya, 2002), and positive functional connectivity of anterior vlPFC and hippocampus is associated with episodic retrieval in humans (Barredo et al., 2015). Understanding how vlPFC-hippocampal coupling influences context encoding and retrieval in the presence of threat is an avenue for future research. 
It is also possible that alterations in hippocampal structure and function due to toxic effects of glucocorticoids (Brunson et al., 2001; Ivy et al., 2010) following childhood violence exposure contributed to general context encoding deficits. These general deficits may have only emerged on angry trials whether hippocampal-dependent contextual processing was greatest in the presence of threat, as previously discussed. Future research is needed to clarify the underlying mechanisms contributing to poor context encoding and memory following childhood violence exposure.

We evaluated a range of potential confounders that might have contributed to individual differences in context memory and neural correlates of context encoding related to violence exposure. Most of these potential confounders were unrelated to behavioral and neural outcomes, although poverty was associated with hippocampal activation and IQ was associated with hippocampal volume and activation. Strong correlations among violence exposure, poverty, and IQ in our sample make it difficult to determine conclusively that the observed associations are truly the result of violence exposure and not poverty or IQ. However, violence exposure was the only factor associated with context memory as well as hippocampal structure, function, and connectivity. Extensive evidence documents attention bias toward angry faces in children exposed to violence (Pollak and Tolley-Schell, 2003; Shackman et al., 2007), consistent with our interpretation that attention bias toward threat interfered with context encoding in children with violence exposure. Nonetheless, future research with larger samples and less overlap among violence exposure, other forms of adversity, and IQ is needed to disentangle their unique influences on context encoding.

Several limitations are worth noting. First, we used realistic pictures of indoor and outdoor scenes appearing in the background of salient facial cues, similar to methods used in adults (Hayes et al., 2007, 2010). However, pictures lack the multisensory experience of real-world contexts. Future research should use ecologically valid contextual stimuli appropriate for an MRI scanner, such as immersive 3D virtual reality (Åhs et al., 2015). Second, we examined neural activation during context encoding, but not context retrieval. Different neural regions underlie context encoding and retrieval in adults (Hayes et al., 2010), highlighting the importance of examining neural correlates of context retrieval developmentally. Third, the study was not designed to examine post-traumatic stress disorder or whether difficulties with context encoding influence later stages of contextual processing relevant to post-traumatic stress disorder, such as context-modulation of fear. Future research should examine whether heightened attention to threat and poor context encoding in the presence of threat contribute to impaired contextmodulation of fear.

In conclusion, context encoding recruits the posterior hippocampus in youth, and hippocampal structure and function are associated with context memory. Context encoding appears to be an implicit process that emerges early in human development. A history of exposure to threatening environments is associated with worse memory for contexts encoded in the presence of threat, which may be explained by smaller hippocampal volume and atypical hippocampal function during context encoding. Future research should examine whether context encoding deficits contribute to persistent fear in safe contexts among children who have been raised in dangerous environments.

\section{References}

Achenbach TM (1991) Integrative guide for the 1991 CBCL/4-18, YSR and TRF profiles. Burlington, VT: Department of Psychiatry, University of Vermont.

Åhs F, Kragel PA, Zielinski DJ, Brady R, LaBar KS (2015) Medial prefrontal pathways for the contextual regulation of extinguished fear in humans. Neuroimage 122:262-271. CrossRef Medline

Anagnostaras SG, Maren S, Fanselow MS (1999) Temporally graded retrograde amnesia of contextual fear after hippocampal damage in rats: within-subjects examination. J Neurosci 19:1106-1114. Medline

Avants BB, Tustison NJ, Song G, Cook PA, Klein A, Gee JC (2011) A reproducible evaluation of ANTs similarity metric performance in brain image registration. Neuroimage 54:2033-2044. CrossRef Medline

Barredo J, Öztekin I, Badre D (2015) Ventral fronto-temporal pathway supporting cognitive control of episodic memory retrieval. Cereb Cortex 25:1004-1019. CrossRef Medline

Barrett LF, Kensinger EA (2010) Context is routinely encoded during emotion perception. Psychol Sci 21:595-599. CrossRef Medline

Beckmann CF, Jenkinson M, Smith SM (2003) General multi-level linear modelling for group analysis in fMRI. Neuroimage 20:1052-1063. CrossRef Medline

Behzadi Y, Restom K, Liau J, Liu TT (2007) A component based noise correction method (CompCor) for BOLD and perfusion based fMRI. Neuroimage 37:90-101. CrossRef Medline

Bernstein DP, Ahluvalia T, Pogge D, Handelsman L (1997) Validity of the Childhood Trauma Questionnaire in an adolescent psychiatric population. J Am Acad Child Adolesc Psychiatry 36:340-348. CrossRef Medline

Bifulco A, Brown GW, Lillie A, Jarvis J (1997) Memories of childhood neglect and abuse: corroboration in a series of sisters. J Child Psychol Psychiatry 38:365-374. CrossRef Medline

Bishop SJ (2008) Neural mechanisms underlying selective attention to threat. Ann N Y Acad Sci 1129:141-152. CrossRef Medline

Brunson KL, Eghbal-Ahmadi M, Bender R, Chen Y, Baram TZ (2001) Longterm, progressive hippocampal cell loss and dysfunction induced by early-life administration of corticotropin-releasing hormone reproduce the effects of early-life stress. Proc Natl Acad Sci U S A 98:8856-8861. CrossRef Medline

Cohen H, Liberzon I, Richter-Levin G (2009) Exposure to extreme stress impairs contextual odour discrimination in an animal model of PTSD. Int J Neuropsychopharmacol 12:291-303. CrossRef Medline

Davachi L (2006) Item, context and relational episodic encoding in humans. Curr Opin Neurobiol 16:693-700. CrossRef Medline

DeMaster DM, Ghetti S (2013) Developmental differences in hippocampal and cortical contributions to episodic retrieval. Cortex 49:1482-1493. CrossRef Medline

Dixon ML, Zelazo PD, De Rosa E (2010) Evidence for intact memoryguided attention in school-aged children. Dev Sci 13:161-169. CrossRef Medline

Eklund A, Nichols TE, Knutsson H (2016) Cluster failure: why fMRI inferences for spatial extent have inflated false-positive rates. Proc Natl Acad Sci U S A 113:7900-7905. CrossRef Medline

Fischl B, Dale AM (2000) Measuring the thickness of the human cerebral cortex from magnetic resonance images. Proc Natl Acad Sci U S A 97: 11050-11055. CrossRef Medline

Ghetti S, Demaster DM, Yonelinas AP, Bunge SA (2010) Developmental differences in medial temporal lobe function during memory encoding. J Neurosci 30:9548-9556. CrossRef Medline

Gogtay N, Nugent TF 3rd, Herman DH, Ordonez A, Greenstein D, Hayashi KM, Clasen L, Toga AW, Giedd JN, Rapoport JL, Thompson PM (2006) Dynamic mapping of normal human hippocampal development. Hippocampus 16:664-672. CrossRef Medline

Hanson JL, Nacewicz BM, Sutterer MJ, Cayo AA, Schaefer SM, Rudolph KD, Shirtcliff EA, Pollak SD, Davidson RJ (2015) Behavioral problems after early life stress: contributions of the hippocampus and amygdala. Biol Psychiatry 77:314-323. CrossRef Medline

Hayes A (2013) Introduction to mediation, moderation, and conditional process analysis. New York: Guilford.

Hayes SM, Nadel L, Ryan L (2007) The effect of scene context on episodic object recognition: parahippocampal cortex mediates memory encoding and retrieval success. Hippocampus 17:873-889. CrossRef Medline

Hayes SM, Baena E, Truong TK, Cabeza R (2010) Neural mechanisms of 
context effects on face recognition: automatic binding and context shift decrements. J Cogn Neurosci 22:2541-2554. CrossRef Medline

Hayne H, Boniface J, Barr R (2000) The development of declarative memory in human infants: age-related changes in deffered imitation. Behav Neurosci 114:77-83. CrossRef Medline

Ivy AS, Rex CS, Chen Y, Dubé C, Maras PM, Grigoriadis DE, Gall CM, Lynch G, Baram TZ (2010) Hippocampal dysfunction and cognitive impairments provoked by chronic early-life stress involve excessive activation of CRH receptors. J Neurosci 30:13005-13015. CrossRef Medline

Jenkinson M, Beckmann CF, Behrens TE, Woolrich MW, Smith SM (2012) Fsl. Neuroimage 62:782-790. CrossRef Medline

Kim JJ, Fanselow MS (1992) Modality-specific retrograde amnesia of fear. Science 256:675-677. CrossRef Medline

Lim L, Radua J, Rubia K (2014) Gray matter abnormalities in childhood maltreatment: a voxelwise metaanalysis. Am J Psychiatry 171:854-863. CrossRef Medline

Maren S, Fanselow MS (1997) Electrolytic lesions of the fimbria/fornix, dorsal hippocampus, or entorhinal cortex produce anterograde deficits in contextual fear conditioning in rats. Neurobiol Learn Mem 67:142-149. CrossRef Medline

McLaughlin KA, Sheridan MA, Lambert HK (2014) Childhood adversity and neural development: deprivation and threat as distinct dimensions of early experience. Neurosci Biobehav Rev 47:578-591. CrossRef Medline

McLaughlin KA, Sheridan MA, Gold AL, Duys A, Lambert HK, Peverill M, Heleniak C, Shechner T, Wojcieszak Z, Pine DS (2016) Maltreatment exposure, brain structure, and fear conditioning in children and adolescents. Neuropsychopharmacology 41:1956-1964. CrossRef Medline

McLaughlin KJ, Gomez JL, Baran SE, Conrad CD (2007) The effects of chronic stress on hippocampal morphology and function: an evaluation of chronic restraint paradigms. Brain Res 1161:56-64. CrossRef Medline

Meulemans T, Van der Linden M, Perruchet P (1998) Implicit sequence learning in children. J Exp Child Psychol 69:199-221. CrossRef Medline

Monk CS, Nelson EE, McClure EB, Mogg K, Bradley BP, Leibenluft E, Blair RJ, Chen G, Charney DS, Ernst M, Pine DS (2006) Ventrolateral prefrontal cortex activation and attentional bias in response to angry faces in adolescents with generalized anxiety disorder. Am J Psychiatry 163:10911097. CrossRef Medline

Monk CS, Telzer EH, Mogg K, Bradley BP, Mai X, Louro HM, Chen G, McClure-Tone EB, Ernst M, Pine DS (2008) Amygdala and ventrolateral prefrontal cortex activation to masked angry faces in children and adolescents with generalized anxiety disorder. Arch Gen Psychiatry 65: 568-576. CrossRef Medline

Ofen N, Chai XJ, Schuil KD, Whitfield-Gabrieli S, Gabrieli JD (2012) The development of brain systems associated with successful memory retrieval of scenes. J Neurosci 32:10012-10020. CrossRef Medline

O’Reilly JX, Woolrich MW, Behrens TE, Smith SM, Johansen-Berg H (2012) Tools of the trade: psychophysiological interactions and functional connectivity. Soc Cogn Affect Neurosci 7:604-609. CrossRef Medline

Petrides M, Pandya DN (2002) Comparative cytoarchitectonic analysis of the human and the macaque ventrolateral prefrontal cortex and corticocortical connection patterns in the monkey. Eur J Neurosci 16:291-310. CrossRef Medline

Phillips RG, LeDoux JE (1992) Differential contribution of amygdala and hippocampus to cued and contextual fear conditioning. Behav Neurosci 106:274-285. CrossRef Medline

Phillips RG, LeDoux JE (1994) Lesions of the dorsal hippocampal formation interfere with background but not foreground contextual fear conditioning. Learn Mem 1:34-44. CrossRef Medline

Pollak SD, Tolley-Schell SA (2003) Selective attention to facial emotion in physically abused children. J Abnorm Psychol 112:323-338. CrossRef Medline

Robinson AJ, Pascalis O (2004) Development of flexible visual recognition memory in human infants. Dev Sci 7:527-533. CrossRef Medline

Roche A (2011) A four-dimensional registration algorithm with application to joint correction of motion and slice timing in fMRI. IEEE Trans Med Imaging 30:1546-1554. CrossRef Medline

Shackman JE, Shackman AJ, Pollak SD (2007) Physical abuse amplifies attention to threat and increases anxiety in children. Emotion 7:838-852. CrossRef Medline

Sheridan MA, McLaughlin KA (2014) Dimensions of early experience and neural development: deprivation and threat. Trends Cogn Sci 18:580585. CrossRef Medline

Shiba Y, Santangelo AM, Roberts AC (2016) Beyond the medial regions of the prefrontal cortex in the regulation of fear and anxiety. Front Syst Neurosci 10:12. CrossRef Medline

Telzer EH, Mogg K, Bradley BP, Mai X, Ernst M, Pine DS, Monk CS (2008) Relationship between trait anxiety, prefrontal cortex, and attention bias to angry faces in children and adolescents. Biol Psychol 79:216-222. CrossRef Medline

Tottenham N, Tanaka JW, Leon AC, McCarry T, Nurse M, Hare TA, Marcus DJ, Westerlund A, Casey BJ, Nelson C (2009) The NimStim set of facial expressions: judgments from untrained research participants. Psychiatry Res 168:242-249. CrossRef Medline

van Marle HJ, Hermans EJ, Qin S, Fernández G (2009) From specificity to sensitivity: how acute stress affects amygdala processing of biologically salient stimuli. Biol Psychiatry 66:649-655. CrossRef Medline

Vinter A, Perruchet P (2000) Implicit learning in children is not related to age: evidence from drawing behavior. Child Dev 71:1223-1240. CrossRef Medline

Vul E, Harris C, Winkielman P, Pashler H (2009) Puzzlingly high correlations in fMRI studies of emotion, personality, and social cognition. Perspect Psychol Sci 4:274-290. CrossRef Medline

Walker EA, Unutzer J, Rutter C, Gelfand A, Saunders K, VonKorff M, Koss MP, Katon W (1999) Costs of health care use by women HMO members with a history of childhood abuse and neglect. Arch Gen Psychiatry 56: 609-613. CrossRef Medline

Wechsler D (1999) Wechsler Abbreviated Scale of Intelligence. San Antonio, TX: Psychological Corporation.

Woolrich M (2008) Robust group analysis using outlier inference. Neuroimage 41:286-301. CrossRef Medline

Woolrich MW, Behrens TE, Beckmann CF, Jenkinson M, Smith SM (2004) Multi-level linear modelling for fMRI group analysis using Bayesian inference. Neuroimage 21:1732-1747. CrossRef Medline

Young SL, Bohenek DL, Fanselow MS (1994) NMDA processes mediate anterograde amnesia of contextual fear conditioning induced by hippocampal damage: immunization against amnesia by context preexposure. Behav Neurosci 108:19-29. CrossRef Medline 